Pacific

Journal of

Mathematics

\title{
ON DUPIN HYPERSURFACES WITH CONSTANT MÖBIUS
} CURVATURE

CARlos M. C. Riveros,

Luciana Avila Rodrigues and Keti Tenenblat

Volume $236 \quad$ No. 1

May 2008 


\title{
ON DUPIN HYPERSURFACES WITH CONSTANT MÖBIUS CURVATURE
}

\author{
Carlos M. C. Riveros, \\ LuCiana Avila Rodrigues AND Keti TENENBLAT
}

\begin{abstract}
We show that proper Dupin hypersurfaces $M^{n}$ for $n \geq 4$ in $\mathbb{R}^{n+1}$ with $n$ distinct principal curvatures and constant Möbius curvature cannot be parametrized by lines of curvature. For $n=3$, up to Möbius transformations, there is a unique proper Dupin hypersurface, parametrized by lines of curvature, with three distinct principal curvatures and constant Möbius curvature. Moreover, these hypersurfaces are the only conformally flat proper Dupin hypersurfaces $M^{3} \subset \mathbb{R}^{4}$ with three distinct principal curvatures and constant Möbius curvature.
\end{abstract}

\section{Introduction}

Dupin surfaces were first studied by Dupin in 1822. In the last three decades, several aspects of Dupin hypersurfaces were studied by many authors [Cecil and Chern 1989; Cecil et al. 2007; Cecil and Jensen 1998; 2000; 1980; Miyaoka 1984; 1989; Niebergall 1991; 1992; Pinkall 1981; 1985b; 1985a; Pinkall and Thorbergsson 1989; Riveros and Tenenblat 2005; Stolz 1999; Thorbergsson 1983]. A hypersurface is said to be Dupin if each principal curvature is constant along its corresponding surface of curvature. A Dupin submanifold $M$ is said to be proper if the number $g$ of distinct principal curvatures is constant on $M$.

The simplest Dupin submanifolds are the isoparametric hypersurfaces, that is, those whose principal curvatures are constant. Such a hypersurface in Euclidean space is an open subset of $\mathbb{R}^{n}$, of the sphere $S^{n}$, or of $S^{k} \times \mathbb{R}^{n-k}$, and hence $g \leq 2$. Cartan classified the isoparametric hypersurfaces of the sphere with $g \leq 3$. Besides isoparametric hypersurfaces, other special Dupin hypersurfaces are those that have constant Möbius curvature or constant Lie curvature.

In this paper, we consider those proper Dupin hypersurfaces of Euclidean space having distinct principal curvatures and constant Möbius curvature.

MSC2000: 53C42, 53A30, 53C40, 53A07.

Keywords: Dupin hypersurfaces, constant Möbius curvature, conformally flat hypersurfaces.

Rodrigues and Tenenblat are partially supported by CNPq, CAPES PROCAD. 
The property of being a proper Dupin hypersurface is invariant under Lie transformations. Therefore the classification of Dupin hypersurfaces is considered up to these transformations. The Möbius (conformal) transformations form a subgroup of the Lie sphere group, and it preserves Möbius curvatures.

Thorbergsson [1983] proved that if $M$ is a proper, Dupin, compact embedded hypersurface then $g=1,2,3,4$, or 6. Pinkall [1985a] showed that there are no restrictions on $g$ if $M$ is not compact. By using basic constructions, namely, tubes, cylinders, and rotational submanifolds, he provided hypersurfaces of the Euclidean space for any $g$. A proper Dupin hypersurface is said to be reducible if it is Lie equivalent to a proper Dupin hypersurface obtained by those basic constructions.

The local classification of Dupin surfaces in $\mathbb{R}^{3}$ says that such a surface is either totally umbilic or it is a Dupin cyclide. Pinkall [1985a] proved that if $M^{n}$ is a Dupin hypersurface of $\mathbb{R}^{n+1}$ and $g=2$, then $M$ is conformally equivalent to an isoparametric hypersurface in $S^{n+1}$. Pinkall [1985b] also gave a complete classification, up to Lie equivalence, for Dupin hypersurfaces $M^{3} \subset \mathbb{R}^{4}$ with three distinct principal curvatures. In particular, he showed that if $M$ is irreducible, then it is locally Lie equivalent to an isoparametric hypersurface of $S^{4}$.

Niebergall [1991; 1992] and more recently Cecil and Jensen [2000] (see also [Cecil et al. 2007]) studied irreducible, proper, Dupin hypersurfaces $M^{4} \subset \mathbb{R}^{5}$ with four distinct principal curvatures. Pinkall [1981] proved that proper Dupin hypersurfaces $M^{n}$ with $g \geq 3$ that are Lie equivalent to isoparametric hypersurfaces cannot be parametrized by lines of curvature.

We will show that those proper Dupin hypersurfaces $M^{n}$ in $\mathbb{R}^{n+1}$ for $n \geq 4$ having $n$ distinct principal curvatures and constant Möbius curvature cannot be parametrized by lines of curvature. For $n=3$, up to Möbius transformations, there is a unique proper Dupin hypersurfaces in $\mathbb{R}^{4}$, parametrized by lines of curvature, having three distinct principal curvatures and constant Möbius curvature.

Moreover, we obtain all the conformally flat Dupin hypersurfaces of $\mathbb{R}^{4}$, with constant Möbius curvature. We recall that for $n \geq 4$, the classical Cartan-Schouten theorem (see [Cartan 1917; Schouten 1921]) proves that $M^{n} \subset \mathbb{R}^{n+1}$ is conformally flat if and only if at least $n-1$ of the principal curvatures coincide at each point. See Chen [1973] for characterizations of such hypersurfaces when $n \geq 4$. For compact conformally flat hypersurfaces, do Carmo, Dajczer, and Mercuri [1985] characterized their topological types, while Pinkall [1988] classified them in the conformal class. Cecil and Ryan [1980] classified conformally flat hypersurfaces with $n \geq 4$ and with the condition of being taut and hence complete. All these results used the Cartan-Schouten theorem, which does not hold in dimension 3. The classification of conformally flat hypersurfaces $M^{3} \subset \mathbb{R}^{4}$ remains open. Such a hypersurface is said to be generic if all the principal curvatures are pairwise 
distinct everywhere. Generic conformally flat hypersurfaces $M^{3} \subset \mathbb{R}^{4}$ with additional assumptions such as constant mean curvature, constant Gauss-Kronecker curvature, or special conditions on the mean curvature vector or the metric were studied by several authors; see [Defever 2000a; 2000b; Garay 1994; Lafontaine 1988; Suyama 2005]. In this paper, we characterize the three dimensional generic conformally flat Dupin hypersurfaces with constant Möbius curvature.

In Section 3, we prove the following result.

Theorem A. Let $M^{n} \subset \mathbb{R}^{n+1}$ for $n \geq 4$ be a proper Dupin hypersurface with $n$ distinct principal curvatures and constant Möbius curvature. Then $M$ cannot be parametrized by lines of curvature.

Theorem A does not hold when we replace Möbius curvatures by Lie curvature. We show in Remark 3.4 that there are Dupin hypersurfaces in $\mathbb{R}^{n+1}$ for $n \geq 4$, parametrized by lines of curvature with $n$ distinct principal curvatures and constant Lie curvature. These hypersurfaces were obtained in [Corro et al. 1999] by using Ribaucour transformations.

For $n=3$, one has this result:

Theorem B. Up to Möbius transformations, there is a unique proper Dupin hypersurface $M^{3}$ that is immersed in $\mathbb{R}^{4}$, parametrized by lines of curvature, and has three distinct principal curvatures and constant Möbius curvature.

Theorem B follows from the classification of Möbius isoparametric hypersurfaces in $S^{4}$ obtained by $\mathrm{Hu}$ and $\mathrm{Li}$ [2005], since one can show that a Dupin hypersurface with constant Möbius curvature is precisely what is called a Möbius isoparametric hypersurface in [ $\mathrm{Li}$ et al. 2002] when there are at least three distinct principal curvatures. However, Theorem B also follows directly from the fundamental theorem for hypersurfaces of the Euclidean space without using the theory of Möbius first and second fundamental forms introduced in [Wang 1998]. This theory was used in [Hu and Li 2005; Hu et al. 2007; Li et al. 2002] to study Möbius isoparametric hypersurfaces of the sphere. Such hypersurfaces are not necessarily Lie equivalent to isoparametric hypersurfaces of the sphere.

The surface of Theorem B is a cone over a flat torus contained on a sphere. It can be parametrized by

$$
X\left(x_{1}, x_{2}, x_{3}\right)=\frac{1}{\sqrt{c} e^{x_{2}}}\left(\frac{\sin x_{1}}{\sqrt{c-1}}, \frac{-\cos x_{1}}{\sqrt{c-1}}, \sin x_{3},-\cos x_{3}\right),
$$

where $c>1$ is a real constant. This is a reducible hypersurface, and it is not Lie equivalent to an isoparametric hypersurface.

We observe that, for Dupin hypersurfaces parametrized by lines of curvature, the condition of having constant Möbius curvature is equivalent to having all higher dimensional Laplace invariants equal to zero; see Lemma 3.2. These invariants 
were introduced by Kamran and Tenenblat [1996; 1998], and they were used to study a class of Dupin hypersurfaces of $\mathbb{R}^{5}$ in [Riveros and Tenenblat 2005].

According to Cartan's theorem [Cartan 1917; Lafontaine 1988] and HertrichJeromin [1994], a conformally flat hypersurface $M^{3} \subset \mathbb{R}^{4}$ with three distinct principal curvatures admits a local coordinate system, called a Guichard net. This is a parametrization by lines of curvature. As a consequence of Theorem B, one gets the following result, which can also be obtained from [Hu and $\mathrm{Li} 2005]$.

Corollary C. Let $M \subset \mathbb{R}^{4}$ be a conformally flat hypersurface with three principal curvatures that are pairwise distinct everywhere. Then $M$ is a Dupin hypersurface with constant Möbius curvature if and only if $M$ is parametrized by $\phi X$, where $\phi$ is a conformal transformation of $\mathbb{R}^{4}$ and $X$ is the cone given by (1).

The cone (1) is a conformally flat hypersurface of hyperbolic type in the class given by Lafontaine [1988].

In Section 2, we include basic results on Dupin hypersurfaces parametrized by lines of curvature and having distinct principal curvatures. In Section 3, we prove Theorem A, and we show that this theorem does not hold if one replaces Möbius curvature by Lie curvature.

\section{Dupin hypersurfaces with distinct principal curvatures}

Let $\Omega$ be a open set of $\mathbb{R}^{n}$ and $x=\left(x_{1}, x_{2}, \ldots, x_{n}\right) \in \Omega$. Let $X: \Omega \subset \mathbb{R}^{n} \rightarrow \mathbb{R}^{n+1}$ be a proper Dupin hypersurface parametrized by lines of curvature with distinct principal curvatures $-\lambda_{i}$ for $1 \leq i \leq n$ and let $N: \Omega \subset \mathbb{R}^{n} \rightarrow \mathbb{R}^{n+1}$ be the unit normal vector field of $X$. Then

$$
\begin{aligned}
\left\langle X_{, i}, X_{, j}\right\rangle & =\delta_{i j} g_{i i}, \\
N_{, i} & =\lambda_{i} X_{, i}, \\
\lambda_{i, i} & =0,
\end{aligned}
$$

where $1 \leq i, j \leq n$ and the subscript ", $i$ " denotes the derivative with respect to $x_{i}$. Moreover,

$$
X_{, i j}-\Gamma_{i j}^{i} X_{, i}-\Gamma_{i j}^{j} X_{, j}=0 \quad \text { for } 1 \leq i \neq j \leq n,
$$

where $\Gamma_{i j}^{k}$ are the Christoffel symbols. Taking the derivative of (3) with respect to $x_{j}$ for $i \neq j$, we have $N_{i j}=\lambda_{i, j} X_{, i}+\lambda_{i} X_{, i j}$. Similarly, $N_{j i}=\lambda_{j, i} X_{, j}+\lambda_{j} X_{, j i}$. Subtracting these equations and substituting $X_{, i j}$ given by (5), it follows that

$$
\Gamma_{i j}^{i}=\frac{\lambda_{i, j}}{\lambda_{j}-\lambda_{i}} \quad \text { for } 1 \leq i \neq j \leq n .
$$


The Christoffel symbols in terms of the metric (2) are given by

$$
\Gamma_{i j}^{k}=0, \quad \Gamma_{i i}^{i}=\frac{g_{i i, i}}{2 g_{i i}}, \quad \Gamma_{i i}^{j}=-\frac{g_{i i, j}}{2 g_{j j}}, \quad \Gamma_{i j}^{i}=\frac{g_{i i, j}}{2 g_{i i}},
$$

where $i, j, k$ are distinct.

For Dupin hypersurfaces with distinct principal curvatures, the Möbius curvature is defined by

$$
C^{i j k}=\frac{\lambda_{i}-\lambda_{j}}{\lambda_{k}-\lambda_{j}} \quad \text { for distinct } i, j, k
$$

Hence, for all distinct $i, j, k$, we have

$$
C^{i j k}=1-C^{i k j}, \quad C^{j i k}=1-\frac{1}{C^{i k j}}, \quad C^{i j k}=\frac{1}{C^{k j i}} .
$$

When $n \geq 4$, we also have

$$
C^{i k j}=C^{i k s} C^{s k j} \quad \text { for distinct } i, j, k, s .
$$

The Lie curvature for $M^{n}$ is a cross ratio of principal curvatures. It is a product of Möbius curvatures defined in [Miyaoka 1989] by

$$
\Psi_{i j k l}=\frac{\left(\lambda_{i}-\lambda_{k}\right)\left(\lambda_{j}-\lambda_{l}\right)}{\left(\lambda_{i}-\lambda_{l}\right)\left(\lambda_{j}-\lambda_{k}\right)} \quad \text { for distinct } i, j, k, l .
$$

For later use, we will obtain some of the properties of the Christoffel symbols and its derivatives and the Gauss equation for Dupin hypersurfaces parametrized by lines of curvature and having distinct principal curvatures.

It follows from (7) that

$$
\begin{aligned}
g_{i i, j} & =2 \Gamma_{i j}^{i} g_{i i} \\
\Gamma_{i i}^{j} & =-\Gamma_{i j}^{i} \frac{g_{i i}}{g_{j j}} .
\end{aligned}
$$

for $1 \leq i \neq j \leq n$. From (6) and (4), we obtain

$$
\Gamma_{i j, i}^{i}=\Gamma_{i j, j}^{j}=\Gamma_{i j}^{i} \Gamma_{i j}^{j} \quad \text { for } 1 \leq i \neq j \leq n .
$$

From (3) and (13) we get

$$
X_{, i i}=\Gamma_{i i}^{i} X_{, i}-\sum_{k \neq i} \Gamma_{i k}^{i} \frac{g_{i i}}{g_{k k}} X_{, k}-\lambda_{i} g_{i i} N .
$$

Using the expressions (7) and (12)-(14), we obtain for $1 \leq i \neq j \leq n$ that

$$
\Gamma_{i i, j}^{j}=\frac{g_{i i}}{g_{j j}}\left(-\Gamma_{i j, j}^{i}+2 \Gamma_{i j}^{i} \Gamma_{j j}^{j}-2\left(\Gamma_{i j}^{i}\right)^{2}\right) .
$$


Proposition 2.1. Let $X: \Omega \subset \mathbb{R}^{n} \rightarrow \mathbb{R}^{n+1}$ for $n \geq 3$ be a proper Dupin hypersurface parametrized by lines of curvature whose principal curvatures $-\lambda_{i}$ for $1 \leq i \leq n$ are distinct. Then the Gauss equation for the immersion $X$ is given by

$$
\lambda_{i} \lambda_{j}+\frac{L_{j i}}{g_{i i}}+\frac{L_{i j}}{g_{j j}}+\sum_{k \neq i \neq j} \frac{\Gamma_{i k}^{i} \Gamma_{j k}^{j}}{g_{k k}}=0
$$

where $i \neq j$ and

$$
L_{j i}=\Gamma_{j i, i}^{i}+\Gamma_{j i}^{j}\left(\Gamma_{j i}^{j}-\Gamma_{i i}^{i}\right)
$$

Proof. For a hypersurface of $\mathbb{R}^{n+1}$, we have the curvature tensor

$$
R_{i j l m}=\sum_{\gamma} g_{i \gamma}\left(\sum_{k}\left(\Gamma_{m j}^{k} \Gamma_{l k}^{\gamma}-\Gamma_{j l}^{k} \Gamma_{k m}^{\gamma}\right)+\Gamma_{m j, l}^{\gamma}-\Gamma_{j l, m}^{\gamma}\right) .
$$

On the other hand, $R_{i j i j}=\lambda_{i} \lambda_{j} g_{i i} g_{j j}$. From the last two equalities, using the fact that the immersion $X$ is a Dupin hypersurface parametrized by lines of curvature, we get

$$
\lambda_{i} \lambda_{j} g_{i i}=\sum_{k}\left(\Gamma_{i i}^{k} \Gamma_{j k}^{j}-\Gamma_{i j}^{k} \Gamma_{i k}^{j}\right)+\Gamma_{i i, j}^{j}-\Gamma_{i j, i}^{j} .
$$

Now it follows from (13) and (16) that

$\lambda_{i} \lambda_{j}+\frac{1}{g_{i i}}\left(\Gamma_{i j, i}^{j}+\Gamma_{i j}^{j}\left(\Gamma_{i j}^{j}-\Gamma_{i i}^{i}\right)\right)+\frac{1}{g_{j j}}\left(\Gamma_{i j, j}^{i}+\Gamma_{i j}^{i}\left(\Gamma_{i j}^{i}-\Gamma_{j j}^{j}\right)\right)+\sum_{k \neq i \neq j} \frac{\Gamma_{i k}^{i} \Gamma_{j k}^{j}}{g_{k k}}=0$.

Therefore we obtain the Gauss equation given by (17).

\section{Dupin hypersurfaces with constant Möbius curvature}

In this section, we prove Theorem A, namely, that proper Dupin hypersurfaces $M^{n}$ in $\mathbb{R}^{n+1}$ for $n \geq 4$ with $n$ distinct principal curvatures and constant Möbius curvature cannot be parametrized by lines of curvature. We also show that this is not true if we replace constant Möbius curvature by constant Lie curvature; see Remark 3.4. We start proving some lemmas.

Lemma 3.1. Suppose $M^{n} \subset \mathbb{R}^{n+1}$ for $n \geq 3$ is a proper Dupin hypersurface that is parametrized by lines of curvature and that has $n$ distinct principal curvatures $-\lambda_{i}$. The Möbius curvatures are constant if and only if, up to a reordering of the 
indices, the principal curvatures are given by

$$
\begin{aligned}
& \lambda_{1}=-(C-1) h_{2}+h_{3}+\sum_{r \geq 4}\left(1-\frac{1}{C_{r}}\right) h_{r}, \\
& \lambda_{2}=\left(1-\frac{1}{C}\right) h_{1}+\frac{1}{C} h_{3}+\sum_{r \geq 4} h_{r}, \\
& \lambda_{3}=h_{1}+h_{2}+\sum_{r \geq 4}\left(1+\frac{1}{C_{r}(C-1)}\right) h_{r}, \\
& \lambda_{s}=C_{s} \lambda_{1}+\left(1-C_{s}\right) \lambda_{2} \quad \text { for } s \geq 4,
\end{aligned}
$$

where $C, C_{s} \in R \backslash\{0,1\}$ for $s \geq 4$ are constants such that $C_{s} \neq 1 /(1-C), C_{r} \neq C_{s}$ for $r \neq s$, and $h_{i}\left(x_{i}\right)$ for $i=1, \ldots, n$ are arbitrary differentiable functions.

Proof. We will first show that each $\lambda_{i}$ is a sum of functions of separated variables. By hypothesis, all Möbius curvatures $C^{i k j}$ are constant, and from (8), we have

$$
\lambda_{i}+\left(C^{i k j}-1\right) \lambda_{k}-C^{i k j} \lambda_{j}=0 \quad \text { for all distinct } i, j, k \text {. }
$$

Differentiating this equation with respect to $x_{k}, x_{j}$, we obtain $\lambda_{i, k j}=0$. Therefore

$$
\lambda_{i}=\sum_{r \neq i} f_{i r}\left(x_{r}\right) \quad \text { for all } i=1, \ldots, n \text {. }
$$

We fix indices $i=1, j=2$, and $k=3$, and we let $C=C^{132}$. Then it follows from (20) and (21) that

$$
\sum_{r \neq 1} f_{1 r}\left(x_{r}\right)+(C-1) \sum_{s \neq 3} f_{3 s}\left(x_{s}\right)-C \sum_{t \neq 2} f_{2 t}\left(x_{t}\right)=0 .
$$

Considering this expression as a sum of functions of distinct variables we have

$$
\begin{array}{ll}
a_{1}=(C-1) f_{31}-C f_{21}, & a_{3}=f_{13}-C f_{23}, \\
a_{2}=f_{12}+(C-1) f_{32}, & a_{r}=f_{1 r}+(C-1) f_{3 r}-C f_{2 r} \quad \text { for } r \geq 4 .
\end{array}
$$

and $\sum_{s=1}^{n} a_{s}=0$. Therefore

$$
\begin{array}{ll}
f_{12}=a_{2}-(C-1) f_{32}, & f_{1 r}=a_{r}-(C-1) f_{3 r}+C f_{2 r} \quad \text { for } r \geq 4, \\
f_{13}=a_{3}+C f_{23}, & f_{21}=(1 / C)\left((C-1) f_{31}-a_{1}\right) .
\end{array}
$$


Letting $a_{1}=a$ in the expression above, it follows from (21) that

$$
\begin{aligned}
& \lambda_{1}=-(C-1) f_{32}+C f_{23}-a+C \sum_{r \geq 4} f_{2 r}-(C-1) \sum_{r \geq 4} f_{3 r}, \\
& \lambda_{2}=\frac{C-1}{C} f_{31}+\frac{1}{C}\left(C f_{23}-a\right)+\sum_{r \geq 4} f_{2 r}, \\
& \lambda_{3}=f_{31}+f_{32}+\sum_{r \geq 4} f_{3 r}, \\
& \lambda_{s}=C_{s} \lambda_{1}+\left(1-C_{s}\right) \lambda_{2}, s \geq 4,
\end{aligned}
$$

where $C_{s}=C^{s 21}=\left(\lambda_{s}-\lambda_{2}\right) /\left(\lambda_{1}-\lambda_{2}\right)$. Since all principal curvatures are distinct, it follows that $C, C_{s} \in R \backslash\{0,1\}$, for all $s \geq 4, C_{s} \neq 1 /(1-C)$, and $C_{r} \neq C_{s}$ for $r \geq 4$ with $r \neq s$.

Now $\lambda_{s}$ does not depend on $x_{s}$; hence $(C-1) f_{3 s}=\left(C-1+1 / C_{s}\right) f_{2 s}-b_{s}$ for all $s \geq 4$, where $b_{s}$ is a constant. Introducing the functions

$$
\begin{array}{ll}
h_{1}\left(x_{1}\right)=f_{31}\left(x_{1}\right), & h_{3}\left(x_{3}\right)=C f_{23}\left(x_{3}\right)-a, \\
h_{2}\left(x_{2}\right)=f_{32}\left(x_{2}\right)-\sum_{s \geq 4} b_{s} /(C-1), & h_{r}\left(x_{r}\right)=f_{2 r}\left(x_{r}\right) \text { for } r \geq 4,
\end{array}
$$

it follows from (22) that the principal curvatures are given by (19).

Conversely, if the principal curvatures are given by (19) then one can see that

$$
\begin{array}{ll}
\lambda_{1}-\lambda_{2}=-\frac{C-1}{C} H, & \lambda_{1}-\lambda_{s}=\frac{\left(C_{s}-1\right)(C-1)}{C} H, \\
\lambda_{1}-\lambda_{3}=-H, & \lambda_{2}-\lambda_{s}=\frac{C_{s}(C-1)}{C} H, \\
\lambda_{2}-\lambda_{3}=-\frac{1}{C} H, & \lambda_{3}-\lambda_{s}=\frac{1+C_{s} C-C_{s}}{C} H,
\end{array}
$$

and $\lambda_{s}-\lambda_{t}=-\left(\left(C_{s}-C_{t}\right)(C-1) / C\right) H$, where

$$
H=h_{1}+C h_{2}-h_{3}+\sum_{r \geq 4} \frac{C}{C_{r}(C-1)} h_{r} .
$$

We conclude that all Möbius curvatures are constant.

The following lemma characterizes Dupin hypersurfaces with constant Möbius curvature in terms of the Christoffel symbols.

Lemma 3.2. Let $M^{n} \subset \mathbb{R}^{n+1}$ for $n \geq 3$ be a proper Dupin hypersurface that is parametrized by lines of curvature and that has $n$ distinct principal curvatures. The Möbius curvature is constant if and only if

$$
\Gamma_{i k}^{i}=\Gamma_{j k}^{j} \quad \text { for all distinct } i, j, k .
$$

Moreover, the Möbius curvature is distinct from 0 and 1. 
Proof. If the Möbius curvature is constant then $\Gamma_{j k}^{j}-\Gamma_{i k}^{i}=\left(\log C^{i k j}\right)_{, k}=0$. Conversely, if (23) holds, then for all distinct $i, j, k$, we have $\left(C^{i j k}\right)_{, j}=0,\left(C^{i k j}\right)_{, k}=0$, and $\left(C^{j i k}\right)_{, i}=0$. It follows from (9) that $C^{i k j}$ does not depend on $x_{i}, x_{k}$, and $x_{j}$. If $n=3$ the theorem is proved. If $n \geq 4$, let $s$ be any index distinct from $i, j, k$; then it follows from (10) that $C^{i k j}=C^{i k s} C^{s k j}$. Since $C^{i k s}$ and $C^{s k j}$ do not depend on $x_{s}$, we conclude that $C^{i k j}$ does not depend on $x_{s}$ for all $s$ distinct from $i, j, k$. Therefore $C^{i k j}$ is constant. Since all $\lambda_{i}$ are distinct, we conclude that $C^{i k j} \neq 0$ and $C^{i k j} \neq 1$.

We observe that the previous lemma shows that constant Möbius curvature is equivalent to having all higher dimensional Laplace invariants equal to zero; see [Kamran and Tenenblat 1996, [1998]] for the definition of these invariants.

We will need the following lemma to prove the main result of this section.

Lemma 3.3. Let $X: \Omega \subset \mathbb{R}^{n} \rightarrow \mathbb{R}^{n+1}$ for $n \geq 3$ be a proper Dupin hypersurface parametrized by lines of curvature such that all the principal curvatures $-\lambda_{r}$ for $1 \leq r \leq n$, are distinct. If all Möbius curvatures are constant then

$$
\sqrt{g_{i i}}\left(\lambda_{j}-\lambda_{i}\right)=F_{j i}\left(x_{i}\right)=C^{j i k} F_{k i}\left(x_{i}\right) \text { for distinct } i, j, k,
$$

where $F_{j i}\left(x_{i}\right)$ is a nonvanishing differentiable function of $x_{i}$. Moreover,

$$
\Gamma_{i i}^{i}=\Gamma_{j i}^{j}+F_{j i}^{\prime} / F_{j i} \quad \text { for } i \neq j,
$$

and the Gauss equation for $i \neq j$ is given by

$$
\lambda_{i} \lambda_{j}+\frac{\lambda_{i}-\lambda_{j}}{F_{j i}^{2}} \tilde{L}_{j i}+\frac{\lambda_{j}-\lambda_{i}}{F_{i j}^{2}} \tilde{L}_{i j}+\sum_{k \neq i \neq j} \frac{\left(\lambda_{i, k}\right)^{2}}{F_{i k}^{2}}=0,
$$

where

$$
\tilde{L}_{j i}=\lambda_{j, i i}+\frac{\left(\lambda_{j, i}\right)^{2}}{\lambda_{i}-\lambda_{j}}-\lambda_{j, i} \frac{F_{j i}^{\prime}}{F_{j i}} .
$$

Proof. It follows from the last equality of (7), that

$$
\frac{\partial}{\partial x_{j}} \log \left(\sqrt{g_{i i}}\left|\lambda_{j}-\lambda_{i}\right|\right)=0 \quad \text { for all } i \neq j .
$$

Hence $\sqrt{g_{i i}}\left(\lambda_{j}-\lambda_{i}\right)=F_{j i}\left(\hat{x_{j}}\right)$, where $F_{j i}\left(\hat{x}_{j}\right)$ is independent of $x_{j}$. It follows that

$$
\frac{F_{j i}\left(\hat{x_{j}}\right)}{\lambda_{j}-\lambda_{i}}=\frac{F_{k i}\left(\hat{x}_{k}\right)}{\lambda_{k}-\lambda_{i}} \quad \text { for all distinct } i, j, k .
$$

Therefore $F_{j i}\left(\hat{x}_{j}\right)=C^{j i k} F_{k i}\left(\hat{x_{k}}\right)$. Since all Möbius curvatures are constant, it follows that $F_{j i}$ depends only on $x_{i}$, and (24) holds. From (7) we conclude that (25) holds. 
The Gauss equation for Dupin hypersurfaces with distinct principal curvatures is given by (17). Observe that

$$
\Gamma_{j i, i}^{j}=\frac{\lambda_{j, i i}}{\lambda_{i}-\lambda_{j}}+\frac{\left(\lambda_{j, i}\right)^{2}}{\left(\lambda_{i}-\lambda_{j}\right)^{2}} .
$$

Hence it follows from (24), (25), and (18) that $\tilde{L}_{j i}=L_{j i}\left(\lambda_{i}-\lambda_{j}\right)$ is given by (27). From Lemma 3.2, we have $\Gamma_{j k}^{j}=\Gamma_{i k}^{i}$ for all distinct $i, j, k$. Using (6) and the equalities above, we conclude that the Gauss equation is given by (26).

We now prove our main result.

Proof of Theorem A. Assume that $M^{n}$ admits a parametrization $X\left(x_{1}, \ldots, x_{n}\right)$ by lines of curvature. Then the principal curvatures $-\lambda_{i}$ satisfy

$$
\left(\lambda_{i} \lambda_{j}\right)_{, r s}=0 \text { for all distinct } i, j, r, s .
$$

In fact, we consider Gauss Equation (26) and take its mixed derivative with respect to $x_{r}$ and $x_{s}$ for $r \neq s$ and distinct from $i$ and $j$. Since from Lemma 3.1, we know that $\lambda_{i, j k}=0$ for all distinct $i, j, k$. We conclude that (28) holds.

Case: $n \geq 5$. We will first show a contradiction in this case. Without loss of generality we can consider $\lambda_{i}$ as in Lemma 3.1 and given by (19) in terms of $n$ functions of one variable $h_{j}\left(x_{j}\right)$. We will prove that the product $h_{A}^{\prime} h_{B}^{\prime}$ equals zero for any pair $1 \leq A \neq B \leq n$ by considering three cases. The first case is $h_{r}^{\prime} h_{s}^{\prime}=0$ for any $r \neq s$ with $r, s \geq 4$. The second is $h_{i}^{\prime} h_{j}^{\prime}=0$ for any $i \neq j$ with $i, j \leq 3$. The third is $h_{i}^{\prime} h_{s}^{\prime}=0$ for any $i \leq 3$ with $s \geq 4$.

Assume that $h_{r}^{\prime} h_{s}^{\prime} \neq 0$ for some $r \neq s$ with $r, s \geq 4$. By considering $\left(\lambda_{1} \lambda_{2}\right)_{, r s}=0$ and $\left(\lambda_{1} \lambda_{3}\right)_{, r s}=0$, we get $\lambda_{2, s} \lambda_{3, r}-\lambda_{2, r} \lambda_{3, s}=0$, which is a contradiction since $C, C_{s} \in R \backslash\{0,1\}$ for $s \geq 4, C_{s} \neq 1 /(1-C)$, and $C_{r} \neq C_{s}$ for $r \neq s$.

Similarly, if $h_{i}^{\prime} h_{j}^{\prime} \neq 0$ for $i \neq j$ and $i, j \leq 3$, then by considering $\left(\lambda_{k} \lambda_{s}\right)_{, i j}=0$ and $\left(\lambda_{k} \lambda_{r}\right)_{, i j}=0$ where $k \leq 3$ is such that $i, j, k$ are distinct and $r \neq s \geq 4$, we obtain a contradiction.

Finally, if $h_{i}^{\prime} h_{s}^{\prime} \neq 0$ for $i \leq 3$ and $s \geq 4$, then by considering $\left(\lambda_{j} \lambda_{k}\right)_{, i s}=0$ and $\left(\lambda_{r} \lambda_{k}\right)_{, i s}=0$ where $j, k \leq 3$ are such that $i, j, k$ are distinct and $r \neq s \geq 4$, we get again a contradiction.

Hence if $n \geq 5$, at most one function $h_{j_{0}}$ is not constant, that is, $h_{i}^{\prime}=0$ for all $i \neq j_{0}$ with $1 \leq i \leq n$. Considering Gauss Equation (26) for the pairs $(i, \ell)$ and $(i, k)$ such that $i, \ell, k, j_{0}$ are distinct, we conclude that

$$
\lambda_{i} \lambda_{\ell}=\lambda_{i} \lambda_{k}=-\frac{\left(\lambda_{i, j_{0}}\right)^{2}}{F_{i j_{0}}^{2}},
$$

which is a contradiction since all principal curvatures are pairwise distinct. 
Therefore if $n \geq 5$, then all functions $h_{j}$ are constant, that is, all principal curvatures are constant. This is a contradiction, since we know that there are no isoparametric hypersurfaces in Euclidean space with $n$ distinct principal curvatures when $n \geq 3$.

Case: $n=4$. This follows from [Hu et al. 2007] and [Pinkall 1981]. However, for the sake of completeness, we will provide a different, simple and direct proof. For $n=4$, since $\lambda_{i}$ are given by (19) in terms of $h_{j}\left(x_{j}\right)$, it follows from (28) that

$$
\begin{array}{rlrl}
{\left[(C+1) C_{4}-1\right] h_{1}^{\prime} h_{2}^{\prime}} & =0, & {\left[(2 C-1) C_{4}+1\right] h_{2}^{\prime} h_{3}^{\prime}} & =0, \\
{\left[(C-2) C_{4}+2\right] h_{1}^{\prime} h_{3}^{\prime}} & =0, & {\left[(C-2) C_{4}+2\right] h_{2}^{\prime} h_{4}^{\prime}} & =0, \\
{\left[(2 C-1) C_{4}+1\right] h_{1}^{\prime} h_{4}^{\prime}} & =0, & {\left[(C+1) C_{4}-1\right] h_{3}^{\prime} h_{4}^{\prime}=0 .}
\end{array}
$$

If $h_{1}^{\prime} h_{2}^{\prime} \neq 0$, then it follows from the left three equations that $C_{4}=1 /(C+1)$, $C+1 \neq 0$, and $h_{3}^{\prime}=h_{4}^{\prime}=0$. Now considering the Gauss equation as in (26) for the pairs of indices $i=3, j=4$ and $i=1, j=3$, we obtain the system of equations

$$
\begin{array}{r}
\lambda_{3} \lambda_{4}+\frac{\left(\lambda_{3,1}\right)^{2}}{\left(F_{31}\left(x_{1}\right)\right)^{2}}+\frac{\left(\lambda_{3,2}\right)^{2}}{\left(F_{32}\left(x_{2}\right)\right)^{2}}=0, \\
\lambda_{1} \lambda_{3}+\frac{\lambda_{1}-\lambda_{3}}{\left(F_{31}\left(x_{1}\right)\right)^{2}} \tilde{L}_{31}+\frac{\left(\lambda_{3,2}\right)^{2}}{\left(F_{32}\left(x_{2}\right)\right)^{2}}=0 .
\end{array}
$$

From (19), we have

$$
\begin{array}{ll}
\lambda_{1}=-(C-1) h_{2}+h_{3}-C h_{4}, & \lambda_{3}=h_{1}+h_{2}+\frac{2 C}{C-1} h_{4}, \\
\lambda_{2}=\left(1-\frac{1}{C}\right) h_{1}+\frac{1}{C} h_{3}+h_{4}, & \lambda_{4}=\frac{C-1}{C+1}\left(h_{1}-h_{2}-\frac{2}{C-1} h_{3}\right) .
\end{array}
$$

Substituting these expressions for $\lambda_{3}$ and $\lambda_{4}$ into Equation (30) and separating variables (recall that $h_{1}$ and $F_{31}$ depend on $x_{1}$, that $h_{2}$ and $F_{32}$ depend on $x_{2}$, and that $h_{3}$ and $h_{4}$ are constant) we get

$$
\begin{gathered}
\frac{C-1}{C+1}\left(h_{1}^{2}+\frac{2}{C-1}\left(h_{3}+C h_{4}\right) h_{1}+\frac{2 C}{(C-1)^{2}} h_{3} h_{4}\right)+\frac{\left(h_{1}^{\prime}\right)^{2}}{F_{31}^{2}}=\frac{A}{C+1}, \\
\frac{C-1}{C+1}\left(-h_{2}^{2}-\frac{2}{C-1}\left(h_{3}-C h_{4}\right) h_{2}+\frac{2 C}{(C-1)^{2}} h_{3} h_{4}\right)+\frac{\left(h_{2}^{\prime}\right)^{2}}{F_{32}^{2}}=-\frac{A}{C+1},
\end{gathered}
$$

where $A$ is a real constant. Therefore we conclude that

$$
F_{31}^{2}=(C+1) \frac{\left(h_{1}^{\prime}\right)^{2}}{D_{31}} \quad \text { and } \quad F_{32}^{2}=(C+1) \frac{\left(h_{2}^{\prime}\right)^{2}}{D_{32}},
$$


where

$$
\begin{aligned}
& D_{31}=-(C-1) h_{1}^{2}-2\left(h_{3}+C h_{4}\right) h_{1}+A-2 C h_{3} h_{4} /(C-1), \\
& D_{32}=(C-1) h_{2}^{2}-2\left(h_{3}-C h_{4}\right) h_{2}-A-2 C h_{3} h_{4} /(C-1),
\end{aligned}
$$

and $A$ is a constant. We substitute these expressions in $\tilde{L}_{31}$, defined by (27) and in (31) and then take the mixed derivative with respect to $x_{1}$ and $x_{2}$. We conclude that $(1-C) h_{1}^{\prime} h_{2}^{\prime}=0$, which is a contradiction.

Similarly, for each fixed pair of functions $h_{i}, h_{j}$ with $i \neq j$ such that $h_{i}^{\prime} h_{j}^{\prime} \neq 0$, we consider in the system (29) those equations that involve $h_{i}^{\prime} h_{j}^{\prime}, h_{i}^{\prime} h_{k}^{\prime}$ and $h_{i}^{\prime} h_{s}^{\prime}$ where $i, j, k, s$ are distinct. Then we get the constant $C_{4}$ given in terms of $C$ and $h_{k}^{\prime}=h_{s}^{\prime}=0$. Now, considering Gauss Equation (26) for the pairs of indices $(k, s)$ and $(i, k)$, we obtain two equations. The first equation will express the functions $F_{k i}$ and $F_{k j}$ in terms of $h_{i}$ and $h_{j}$, and the second equation will give $h_{i}^{\prime} h_{j}^{\prime}=0$, which is a contradiction.

Remark 3.4. Theorem A does not hold when we replace Möbius curvature by Lie curvature. We observe that there are Dupin hypersurfaces in $\mathbb{R}^{n+1}$ for $n \geq 4$ that are parametrized by lines of curvature and have $n$ distinct principal curvatures and constant Lie curvature. In what follows, we will exhibit such Dupin hypersurfaces in $\mathbb{R}^{n+1}$. These hypersurfaces were obtained in [Corro et al. 1999] by applying Ribaucour transformations to a hyperplane. Consider the hypersurface in the Euclidean space $\mathbb{R}^{n+1}$ parametrized by

$$
X\left(x_{1}, \ldots, x_{n}\right)=\left(x_{1}, \ldots, x_{n}, 0\right)-\frac{2 \sum_{j=1}^{n} f_{j}}{\sum_{j=1}^{n}\left(f_{j}^{\prime}\right)^{2}+b^{2}}\left(f_{1}^{\prime}, \ldots, f_{n}^{\prime},-b\right) .
$$

Then $X$ is a parametrized Dupin hypersurface, where $\left(x_{1}, \ldots, x_{n}\right) \in U \subset \mathbb{R}^{n}$ and

$$
f_{j}=b_{j 2} x_{j}^{2}+b_{j 1} x_{j}+b_{j 0} \quad \text { for } b \neq 0, b_{j 2}, b_{j 1}, b_{j 0} \in R \text { and } 1 \leq j \leq n \text {. }
$$

The principal curvatures of $X$ are given by

$$
-\lambda_{l}=\frac{4 b b_{l 2}}{D_{l}} \quad \text { for } 1 \leq l \leq n,
$$

where

$$
\begin{aligned}
D_{l} & =\sum_{j \neq l}^{n}\left(f_{j}^{\prime}\right)^{2}-4 b_{l 2} \sum_{j \neq l}^{n} f_{j}+a_{l}, \\
a_{l} & =b^{2}+b_{l 1}^{2}-4 b_{l 2} b_{l 0} .
\end{aligned}
$$


By introducing the notation

$$
S_{1}=\sum_{r=1}^{n}\left(f_{r}^{\prime}\right)^{2} \quad \text { and } \quad S=\sum_{r=1}^{n} f_{r}
$$

expression (34) reduces to $D_{l}=S_{1}-\left(f_{l}^{\prime}\right)^{2}-4 b_{l 2}\left(S-f_{l}\right)+a_{l}$. Using (32) and (35), we have $-\left(f_{l}^{\prime}\right)^{2}+4 b_{l 2} f_{l}+a_{l}=b^{2}$. Hence for each $l$ we have

$$
D_{l}=S_{1}-4 b_{l 2} S+b^{2} \text {. }
$$

It follows from (33) that

$$
\lambda_{i}-\lambda_{k}=\frac{4 b\left(b_{k 2}-b_{i 2}\right)\left(S_{1}+b^{2}\right)}{D_{i} D_{k}} .
$$

If the coefficients $b_{j 2}$ are distinct for all $j$, the principal curvatures have multiplicity one. The Möbius curvatures

$$
C^{i k l}=\frac{\lambda_{i}-\lambda_{k}}{\lambda_{l}-\lambda_{k}}=\frac{\left(b_{k 2}-b_{i 2}\right) D l}{\left(b_{k 2}-b_{l 2}\right) D_{i}}
$$

are rational functions, and the Lie curvatures given by (11) are constant

$$
\Psi_{i j k l}=\frac{\left(b_{i 2}-b_{k 2}\right)\left(b_{j 2}-b_{l 2}\right)}{\left(b_{i 2}-b_{l 2}\right)\left(b_{j 2}-b_{k 2}\right)} .
$$

\section{References}

[do Carmo et al. 1985] M. do Carmo, M. Dajczer, and F. Mercuri, "Compact conformally flat hypersurfaces”, Trans. Amer. Math. Soc. 288:1 (1985), 189-203. MR 86b:53052 Zbl 0537.53050

[Cartan 1917] E. Cartan, "La déformation des hypersurfaces dans l'espace conforme réel à $n \geq 5$ dimensions”, Bull. Soc. Math. France 45 (1917), 57-121. MR 1504762 JFM 46.1129.02

[Cecil and Chern 1989] T. E. Cecil and S. S. Chern, "Dupin submanifolds in Lie sphere geometry", pp. 1-48 in Differential geometry and topology (Tianjin, 1986-87), edited by B. J. Jiang et al., Lecture Notes in Math. 1369, Springer, Berlin, 1989. MR 90i:53079 Zbl 0678.53003

[Cecil and Jensen 1998] T. E. Cecil and G. R. Jensen, "Dupin hypersurfaces with three principal curvatures”, Invent. Math. 132:1 (1998), 121-178. MR 2000k:53051 Zbl 0908.53007

[Cecil and Jensen 2000] T. E. Cecil and G. R. Jensen, "Dupin hypersurfaces with four principal curvatures”, Geom. Dedicata 79:1 (2000), 1-49. MR 2001g:53103 Zbl 0965.53039

[Cecil and Ryan 1980] T. E. Cecil and P. J. Ryan, "Conformal geometry and the cyclides of Dupin", Canad. J. Math. 32:4 (1980), 767-782. MR 82f:53004 Zbl 0483.53050

[Cecil et al. 2007] T. E. Cecil, Q.-S. Chi, and G. R. Jensen, "Dupin hypersurfaces with four principal curvatures, II", Geom. Dedicata 128 (2007), 55-95. MR 2350147 Zbl 05244754

[Chen 1973] B.-y. Chen, Geometry of submanifolds, Marcel Dekker, New York, 1973. Pure and Applied Mathematics, No. 22. MR 50 \#5697 Zbl 0262.53036

[Corro et al. 1999] A. V. Corro, W. Ferreira, and K. Tenenblat, "On Ribaucour transformations for hypersurfaces”, Mat. Contemp. 17 (1999), 137-160. MR 2002f:53104 Zbl 1018.53004 
[Defever 2000a] F. Defever, "Conformally flat hypersurfaces of $\mathbf{E}^{4}$ with constant mean curvature", Results Math. 37:3-4 (2000), 322-330. MR 2001a:53093 Zbl 0980.53007

[Defever 2000b] F. Defever, "Conformally flat hypersurfaces with constant Gauss-Kronecker curvature”, Bull. Austral. Math. Soc. 61:2 (2000), 207-216. MR 2001b:53068 Zbl 0978.53009

[Garay 1994] O. J. Garay, "A classification of certain 3-dimensional conformally flat Euclidean hypersurfaces”, Pacific J. Math. 162:1 (1994), 13-25. MR 94m:53078 Zbl 0791.53026

[Hertrich-Jeromin 1994] U. Hertrich-Jeromin, “On conformally flat hypersurfaces and Guichard's nets”, Beiträge Algebra Geom. 35:2 (1994), 315-331. MR 95j:53022 Zbl 0820.53003

[Hu and Li 2005] Z. Hu and H. Li, "Classification of Möbius isoparametric hypersurfaces in $\mathbb{S}^{4}$ ", Nagoya Math. J. 179 (2005), 147-162. MR 2006e:53098 Zbl 05010498

[Hu et al. 2007] Z. Hu, H. Li, and C. Wang, "Classification of Möbius isoparametric hypersurfaces in S5", Monatsh. Math. 151:3 (2007), 201-222. MR 2329083 Zbl 05180833

[Kamran and Tenenblat 1996] N. Kamran and K. Tenenblat, "Laplace transformation in higher dimensions”, Duke Math. J. 84:1 (1996), 237-266. MR 97k:58181 Zbl 0857.53004

[Kamran and Tenenblat 1998] N. Kamran and K. Tenenblat, "Periodic systems for the higherdimensional Laplace transformation", Discrete Contin. Dynamical Systems 4:2 (1998), 359-378. MR 98m:35139 Zbl 0958.35088

[Lafontaine 1988] J. Lafontaine, "Conformal geometry from the Riemannian viewpoint", pp. 65-92 in Conformal geometry (Bonn, 1985/1986), edited by R. S. Kulkarni and U. Pinkall, Aspects Math., E12, Vieweg, Braunschweig, 1988. MR 90a:53022 Zbl 0661.53008

[Li et al. 2002] H. Z. Li, H. L. Liu, C. P. Wang, and G. S. Zhao, "Möbius isoparametric hypersurfaces in $\mathbf{S}^{n+1}$ with two distinct principal curvatures", Acta Math. Sin. (Engl. Ser.) 18:3 (2002), 437-446. MR 2003h:53079 Zbl 1030.53017

[Miyaoka 1984] R. Miyaoka, "Compact Dupin hypersurfaces with three principal curvatures", Math. Z. 187:4 (1984), 433-452. MR 85h:53041 Zbl 0529.53045

[Miyaoka 1989] R. Miyaoka, "Dupin hypersurfaces and a Lie invariant”, Kodai Math. J. 12:2 (1989), 228-256. MR 90i:53083 Zbl 0687.53053

[Niebergall 1991] R. Niebergall, "Dupin hypersurfaces in $\mathbf{R}^{5}$, I", Geom. Dedicata 40:1 (1991), 122. MR 92k:53106 Zbl 0733.53031

[Niebergall 1992] R. Niebergall, "Dupin hypersurfaces in $\mathbf{R}^{5}$. II", Geom. Dedicata 41:1 (1992), 5-38. MR 93b:53008

[Pinkall 1981] U. Pinkall, Dupin'sche Hyperflachen, PhD thesis, Univertität Freiburg, 1981.

[Pinkall 1985a] U. Pinkall, "Dupin hypersurfaces”, Math. Ann. 270 (1985), 427-440. MR 86e:53044 Zbl 0538.53004

[Pinkall 1985b] U. Pinkall, "Dupin'sche Hyperflächen in $E^{4}$,, Manuscripta Math. 51:1-3 (1985), 89-119. In German. MR 86m:53010 Zbl 0572.53028

[Pinkall 1988] U. Pinkall, “Compact conformally flat hypersurfaces”, pp. 217-236 in Conformal geometry (Bonn, 1985/1986), edited by R. S. Kulkarni and U. Pinkall, Aspects Math., E12, Vieweg, Braunschweig, 1988. MR 90a:53071 Zbl 0657.53030

[Pinkall and Thorbergsson 1989] U. Pinkall and G. Thorbergsson, "Deformations of Dupin hypersurfaces”, Proc. Amer. Math. Soc. 107:4 (1989), 1037-1043. MR 90c:53145 Zbl 0682.53061

[Riveros and Tenenblat 2005] C. M. C. Riveros and K. Tenenblat, "Dupin hypersurfaces in $\mathbb{R}^{5}$ ", Canad. J. Math. 57:6 (2005), 1291-1313. MR 2006i:53089 Zbl 05008894 
[Schouten 1921] J. A. Schouten, "Über die konforme Abbildung n-dimensionaler Mannigfaltigkeiten mit quadratischer Maßbestimmung auf eine Mannigfaltigkeit mit euklidischer Maßbestimmung", Math. Z. 11:1-2 (1921), 58-88. MR 1544484 Zbl 48.0857.02

[Stolz 1999] S. Stolz, "Multiplicities of Dupin hypersurfaces", Invent. Math. 138:2 (1999), 253-279. MR 2001d:53065 Zbl 0944.53035

[Suyama 2005] Y. Suyama, "Conformally flat hypersurfaces in Euclidean 4-space, II", Osaka J. Math. 42:3 (2005), 573-598. MR 2006g:53084 Zbl 1085.53052

[Thorbergsson 1983] G. Thorbergsson, "Dupin hypersurfaces”, Bull. London Math. Soc. 15 (1983), 493-498. MR 85b:53066 Zbl 0529.53044

[Wang 1998] C. Wang, "Moebius geometry of submanifolds in $S^{n}$ ", Manuscripta Math. 96:4 (1998), 517-534. MR 2000a:53019 Zbl 0912.53012

Received June 13, 2007. Revised February 22, 2008.

Carlos M. C. Riveros

Departamento de Matemática

UNIVERSIDADE DE BRASÍlIA

70910-900, BRASÍLIA, DF

BRAZIL

carlos@mat.unb.br

LuCiana AVILA Rodrigues

Instituto de MATEMÁticA E Estatística

UNIVERSIDADE FEDERAL DE GOIÁS

74001-970, GoIÂNIA, GO

BRAZIL

luavila@mat.ufg.br

Keti Tenenblat

Departamento de Matemática

UNIVERSIDADE DE BRASÍLIA

70910-900, BRASÍLIA, DF

BRAZIL

keti@mat.unb.br 